\title{
Use Of Microwave Processing In The Analysis Of Mitochondrial Ultrastructure Injury
}

\author{
G.D. Josephsen*, K.A. Josephsen**, G.J. Beilman, MD***, J. Taylor, MD***, K.E. Muiler, \\ MBS*** \\ *University of Minnesota, School of Medicine, 420 Delaware St. SE, MMC 293, Minneapolis, MN \\ 55455 \\ **Imaging Center, University Of Minnesota, 1475 Gortner Ave. St. Paul, MN 55117 \\ ***Division of Surgical Critical Care, Fairview University Medical Center, 420 Delaware St. S.E., \\ Minneapolis, MN 55455
}

Due to the central role that mitochondria play in numerous disease states, analysis of their injury status has become an important tool in medical research. The degree of injury can be qualitatively ascertained by recognizing changes in morphology during injury, and assigning a score (0-5) based on the cellular injury scale [3]. Our interest lies in the ultrastuctural damage incurred by models of shock, for which this method of mitochondrial ultrastucture injury scoring has been well established [1][2]. Problems with this type of investigation include fixation of mitochondria in tissue samples and long turn-around time for processing samples for transmission electron microscopy (TEM). We have circumvented these problems by using a protocol adapted from previous microwave processing techniques which involves immediate post-excision fixation in a glutaraldehyde fixative using a PELCO ${ }^{\circledR}$ BioWave ${ }^{\mathrm{TM}}$ (TED PELLA, INC.). By reducing processing times we were allowed increased efficiency in the lab without sacrificing the ultrastructural detail necessary for our analysis. A porcine model for hemorrhagic shock was created by inducing a $35 \%$ bleed via IVC cannula followed by reperfusion for 90 minutes. Hepatic biopsies $2 \times 2 \mathrm{~cm}$ in size were biopsied (before and after shock), immersed directly into a gluteraldehyde fixative, then cut into $0.5 \mathrm{~mm}$ cubes and placed into fresh fixative for microwaving. This was carried out within 2 minutes of biopsy. The irradiation in our Biowave was 4 minutes at 309 watts under vacuum followed by a two minute rest and another 4 minutes under vacuum at 309 watts (mic:4x2x4@309W +vac -- note: all microwave steps were carried out at 25 degrees celsius). Use of the microwave in the preliminary steps normalizes the level of fixation between tissue pieces that vary in size, decreasing system error in ultrastructural analysis of volatile structures such as mitochondria. Samples were processed and sectioned onto copper grids, randomized, and photographed under TEM (Philips CM12) at 22,000x. The negatives were scanned as 240 dpi tiff files, and viewed for mitochondrial scoring by an observer blind to their randomization, using the Mitochondrial Injury Scoring System (Table 1). Samples pre- and postshock of each of the two animals were photographed in 10 locations each containing 5-12 mitochondria (total $n=258$ ). Results showed evidence of increased ultrastructural damage in the post-shock samples versus their pre-shock controls (see figure 1 and 2) similar to that seen in a model of gram-negative septic shock [1][2]. These methods of preparation have proved effective in the time-efficient processing of specimens for TEM without sacrificing the detail necessary for ultrastructural analysis. Future investigations will involve coupling this technique with digital analysis developed by Crouser et al. for fast objective ascertainment of the level of mitochondrial damage based on morphology [6].

References 
[1] E.D.Crouser et al., Am. J. Respir. Crit. Care Med. 160 (1999), 1347-1353

[2] E.D.Crouser et al., Am. J. Respir. Crit. Care Med. 161 (2000), 1705-1712

[3] B.F. Trump et al., Sca. Electron. Microsc. 62 (1980), 437-492

[4] Giberson et al., J. Vet. Diag. Invest. 9 (1997), 61-67

[5] Giberson et al., unpublished

[6] E.D Crouser et al., Analyt. Cell. Path. 21 (2000), 41-48

[7] This research was supported by Office of Naval Research, \# N000140210093.

Figure 1. TEM of hepatic mitochondrial pre-shock (A) and post-shock (B, C, and D) which show examples of ultrastructural damage.
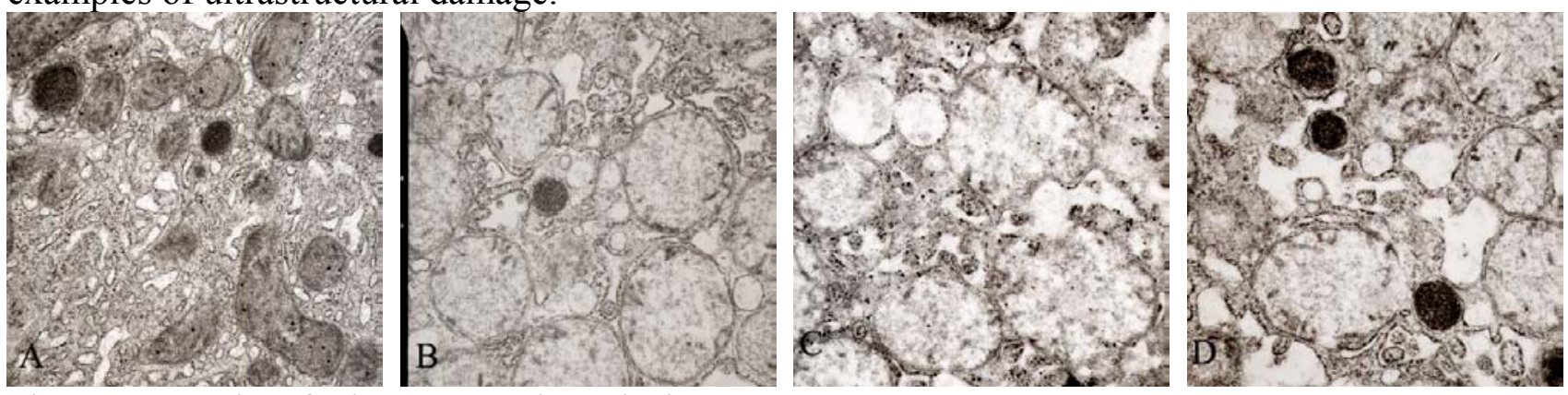

Figure 2. Results of Ultrastructural Analysis

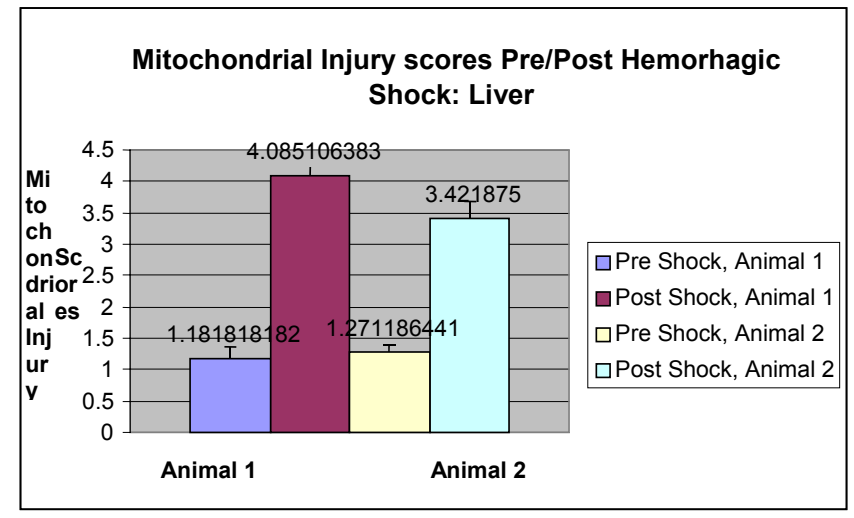

Table 1. Mitochondrial Injury Scoring System. Adapted from Crouser, et al.

\begin{tabular}{|l|l|l|}
\hline Injury Stage & Score & Characteristics \\
\hline I & 0.0 & $\begin{array}{l}\text { Normal appearance, normal mitochondrial granules, other organelles in normal } \\
\text { relationship. Loss of granules and reduction in glycogen. }\end{array}$ \\
\hline II & 1.0 & $\begin{array}{l}\text { Swelling of ER/ Dilation of cisternae, minimal mitochondrial swelling, cell surface } \\
\text { blebs, condensation/shrinkage of inner mitochondrial compartment and enlargement of } \\
\text { intra-crystal space. RER is dilated. }\end{array}$ \\
\hline III & 2.0 & $\begin{array}{l}\text { Mild mitochondrial swelling. Some with high amplitude inner compartment swelling } \\
\text { and other only condensed. }\end{array}$ \\
\hline $\mathrm{IV}_{\mathrm{a}}$ & 3.0 & All mitochondria swollen and inner compartments expanded. \\
\hline $\mathrm{IV}_{\mathrm{b}}$ & 4.0 & Not all swollen but some contain tiny dense aggregates. \\
\hline IV & 5.0 & Massive swelling with flocculent densities, calcification, and vesiculations form. \\
\hline $\mathrm{V} / \mathrm{VI}$ & &
\end{tabular}

